\title{
Orientation Selectivity in Macaque V1: Diversity and Laminar Dependence
}

\author{
Dario L. Ringach, ${ }^{1}$ Robert M. Shapley, ${ }^{2}$ and Michael J. Hawken ${ }^{2}$ \\ ${ }^{1}$ Department of Neurobiology, David Geffen School of Medicine, Department of Psychology and Brain Research Institute, \\ University of California, Los Angeles, Los Angeles, California 90095, and ${ }^{2}$ Center for Neural Science, New York University, \\ New York, New York 10003
}

\begin{abstract}
We studied the steady-state orientation selectivity of single neurons in macaque primary visual cortex (V1). To analyze the data, two measures of orientation tuning selectivity, circular variance and orientation bandwidth, were computed from the tuning curves. Circular variance is a global measure of the shape of the tuning curve, whereas orientation bandwidth is a local measure of the sharpness of the tuning curve around its peak. Circular variance in V1 was distributed broadly, indicating a great diversity of orientation selectivity. This diversity was also reflected in the individual cortical layers. However, there was a tendency for neurons with high circular variance, meaning low selectivity for orientation, to be concentrated in layers 4C, 3B, and 5 . The relative variation of orientation bandwidth across the cortical layers was less than for circular variance, but it showed a similar laminar dependence. Neurons with large orientation
\end{abstract}

Selectivity for the orientation of a visual stimulus is an emergent property of neurons in the primary visual cortex (V1) (Hubel and Wiesel, 1962, 1968). The mechanisms of this selectivity are still debated (for review, see, Sompolinsky and Shapley, 1997; Ferster and Miller, 2000). The functional role of orientation selectivity and its mechanisms in the primate brain would be clearer if we knew how orientation selectivity varies among different populations of V1 neurons and throughout V1 layers. Previously, there were studies of the distribution of orientation bandwidth (Schiller et al., 1976; DeValois et al., 1982) and its laminar distribution (Schiller et al., 1976) in macaque V1. However, theories of the neural mechanisms of orientation selectivity in V1 are concerned with the suppression of responses far from the preferred orientation. Therefore, to obtain results on orientation selectivity of the V1 population that could be compared with theory, it was necessary to use a more global measure of selectivity than had been used in previous studies of macaque V1.

Our experiments measured steady-state orientation selectivity with achromatic black-white sine gratings on a large population of V1 neurons of known laminar location in V1. Orientationtuning selectivity was estimated with two different quantitative measures: circular variance and bandwidth. The circular variance of the response is a global measure (Mardia, 1972; Batschelet,

\footnotetext{
Received March 18, 2002; revised April 19, 2002; accepted April 22, 2002.

This work was supported by National Institutes of Health Grants EY-12816 (D.L.R.), EY-08300 (M.J.H.), and EY-01472 (R.M.S.).

Correspondence should be addressed to Dr. Dario Ringach, Departments of Neurobiology and Psychology, Franz Hall, Room 8441B, University of California, Los Angeles, Los Angeles, CA 90095-1563. E-mail: dario@ucla.edu.

Copyright (C) 2002 Society for Neuroscience $0270-6474 / 02 / 225639-13 \$ 15.00 / 0$
}

bandwidth were found predominantly in layers $4 \mathrm{C}$ and 3B. There was a weak correlation between orientation selectivity and the level of spontaneous activity of the neurons. We also assigned a response modulation ratio for each cell, which is a measure of the linearity of spatial summation. Cells with low modulation ratios tended to have higher circular variance and bandwidth than those with high modulation ratios. These findings suggest a revision to the classical view that nonoriented receptive fields are principally found in layer $4 \mathrm{C}$ and the cytochrome oxidase-rich blobs in layer 2/3. Instead, a broad distribution of tuning selectivity is found in all cortical layers, and neurons that are weakly tuned for orientation are ubiquitous in V1 cortex.

Key words: primate vision; striate cortex; orientation selectivity; cortical layer; circular variance; bandwidth
1981; Swindale, 1998). One of our main findings is that there is a great diversity of circular variance in V1.

The half-bandwidth at some criterion height (we used $1 / \sqrt{2}$ height of the maximum response following Schiller et al., 1976) is a local measure of tuning around the preferred orientation. It might be the case that the diversity in circular variance is directly caused by the neural mechanisms that cause variation in bandwidth. However, the data indicate that circular variance and bandwidth are not so simply related. The data do not agree with a simple one-parameter model (see Appendix) that can describe the shape of all tuning curves. Besides bandwidth, the shape of the tuning curve far from the preferred orientation has a strong influence on circular variance.

We also studied the dependence of orientation selectivity on the linearity of neural signal summation, as estimated by the modulation ratio $M=R(F 1) / R(F 0)$, where $R(F 1)$ is the fundamental response, and $R(F 0)$ is the DC response to drifting sine gratings (Skottun et al., 1991). However, it was noted recently that the observed bimodality of the modulation ratio could occur in a network with unimodally distributed physical parameters (Mechler and Ringach, 2002). Therefore, instead of studying the orientation selectivity of cells separated into simple and complex classes, we studied the correlation between measures of orientation selectivity and the (continuously varying) modulation ratio. There was a significant correlation of circular variance, but not bandwidth, with the modulation ratio. This suggests that the mechanisms that affect the modulation ratio may also influence circular variance.

Laminar variation of orientation selectivity may be a clue to mechanisms because functional connectivity varies in different 
V1 layers. There were noticeable trends in the laminar variation of circular variance and bandwidth, but these were not statistically significant because of the wide diversity within each layer. We also found variation in the modulation ratio across the cortical laminas, with the highest median modulation ratio in the input layers $4 C \alpha$ and $\beta$.

\section{MATERIALS AND METHODS}

Preparation and recording. Acute experiments were performed on 26 adult Old World monkeys (Macaca fascicularis) weighing between 2.5 and $5.1 \mathrm{~kg}$. The methods of preparation and single-cell recording are the same as those described by Sceniak et al. (2001). Animals were tranquilized with $50 \mu \mathrm{g} / \mathrm{kg}$ acepromazine intramuscularly, anesthetized with ketamine (30 mg/kg, i.m.), and maintained on intravenous opioid anesthetic (sufentanil citrate; $6 \mu \mathrm{g} \cdot \mathrm{kg}^{-1} \cdot \mathrm{hr}^{-1}$ ) for the surgery. For recording, anesthesia was continued with $6 \mu \mathrm{g} \cdot \mathrm{kg}^{-1} \cdot \mathrm{hr}^{-1}$ sufentanil, and paralysis was induced with pancuronium bromide $\left(0.1-0.2 \mathrm{mg} \cdot \mathrm{kg}^{-1} \cdot \mathrm{hr}^{-1}\right)$. Electrocardiogram, EEG, and end-tidal $\mathrm{CO}_{2}$ were continuously monitored. Blood pressure was measured non-invasively at $5 \mathrm{~min}$ intervals. Body temperature was maintained at $37^{\circ} \mathrm{C}$. Extracellular action potentials were recorded with glass-coated tungsten microelectrodes, with 5-15 $\mu \mathrm{m}$ exposed tips (Merrill and Ainsworth, 1972). Electrical signals were amplified in the conventional manner, and spikes were discriminated using a two-channel window sorter, which generated TTL pulses that were accumulated as event times by the computer (with $1 \mathrm{msec}$ accuracy). Strict criteria for single-unit recording included the following: fixed nerve impulse height and waveform and absence of impulse intervals shorter than an absolute refractory period. In most of the experiments described here, data were collected by a Cambridge Electronics Design (Cambridge, UK) 1401+ laboratory interface connected to a personal computer. Stimuli were generated on a Silicon Graphics (Mountain View, CA) Elan R4000 or O2 computer and displayed on a Barco (Kortrijk, Belgium) CCID 7651 monitor at a refresh rate of 60 or $120 \mathrm{~Hz}$ (Ringach et al., 1997) or on a Sony (Tokyo, Japan) 502 monitor at a refresh rate of $100 \mathrm{~Hz}$. For all displays, the mean luminance was between 55 and $65 \mathrm{~cd} / \mathrm{m}^{2}$. The displays were calibrated and linearized by lookup tables. A Photo Research (Chatsworth, CA) model 703-PC spectroradiometer was used to calibrate the display screens.

Histology. Three to six electrolytic lesions $(2-3 \mu \mathrm{A}$ for $2-3 \mathrm{sec}$, tip negative) were made along the length of each electrode penetration. To improve the accuracy of laminar localization, the electrode was angled obliquely with respect to the cortical surface. The angle of the electrode track, relative to the normal to the surface of the cortex, was approximately $60^{\circ}$. A typical electrode track would extend for $\sim 4-5 \mathrm{~mm}$. Consecutive lesions were spaced by $\sim 1 \mathrm{~mm}$. Some intentional variation in the distances between neighboring lesions was imposed to ease in the identification of the lesions during the reconstruction. In cases in which not all lesions could be recovered, we discarded the data. For the data in this paper, we made 46 penetrations, obtaining acceptable data on average from approximately seven cells along each electrode track. Our electrode tracks resembled the one shown by Hawken and Parker (1984). At the end of the experiment, the animal was killed by an overdose of anesthetic and perfused through the heart. The details of fixation, sectioning, staining, and reconstruction of electrode tracks are described in detail by Hawken et al. (1988). Subdividing layer $4 \mathrm{C}$ into $4 \mathrm{C} \alpha$ and $4 \mathrm{C} \beta$ with $4 \mathrm{C} \alpha$ represented by the top one-third of $4 \mathrm{C}$ is based on the labeling of the afferents from the magnocellular and parvocellular layers of the lateral geniculate nucleus (LGN), which are segregated in 4C (Hendrickson et al., 1978; Lund, 1988). Our analyses do not depend on the exact location of this boundary, which we include in the graphs only as a reference. It has been suggested to us that the border between $4 \mathrm{C} \alpha$ and $4 \mathrm{C} \beta$ lies more toward the middle of layer 4C (E. Callaway, personal communication). Layer $3 \mathrm{~B}$ is defined as the region receiving projections from spiny stellate cells in layer 4C. Estimates of the relative width of layer 3B compared with 2/3 range between 33 and $40 \%$ (Blasdel and Fitzpatrick, 1984; Fitzpatrick et al., 1985; Lund, 1988; Lachica et al., 1992; Yabuta and Callaway, 1998). We chose our dividing line in the middle of this range, with layer $3 \mathrm{~B}$ taking the bottom $37 \%$ of the entire $2 / 3$ width.

We are confident that our reconstruction method does not incur significant errors or biases. For example, our localization of directional cells in layers 4B and 6 (Hawken et al., 1988) and color cells in 4C $\beta$ (Johnson et al., 2001) agrees very well with previous studies. On many occasions, we moved the electrode $>1 \mathrm{~mm}$ away from a spatially re- stricted cluster of cells with some specific visual properties and were able to move the electrode back to the same location just based on the micromanipulator reading. Thus, we do not suspect any significant dragging of the tissue by the electrode. Also, the fact that we observe sharp transitions in the response properties of cells as a function of normalized cortical depth, even after pooling data across animals (see Results), suggests that the method was applied consistently across animals.

Finally, we encountered difficulties obtaining good visualization of the cytochrome oxidase (CO) blobs in layer $2 / 3$. The data we have currently are not sufficient to analyze the dependence of tuning in blob and interblob regions in layer $2 / 3$. However, we made use of CO-stained tissue to help us define the boundary between $4 \mathrm{~B}$ and $4 \mathrm{C} \alpha$.

Procedures. Each cell was stimulated monocularly via the dominant eye and characterized by measuring its steady-state response to conventional drifting sinusoidal gratings (the nondominant eye was occluded). With this method, we measured basic attributes of the cell, including spatial and temporal frequency tuning, orientation tuning, contrast response function, and color sensitivity, as well as area, length, and width tuning curves.

The spatial and temporal frequencies used during the measurement of a steady-state orientation tuning curve were chosen to be optimal for the cell. Steady-state orientation tuning curves were obtained using angular steps of 15 or $20^{\circ}$. In a few very sharply tuned cells, we used steps of $10^{\circ}$. The response at each orientation was averaged for $4 \mathrm{sec}$, over 8-64 grating temporal periods $(2-16 \mathrm{~Hz}$ drift rate, depending on the optimal temporal frequency of the neuron).

Each grating stimulus was presented through a circular window with sharp edges. The mean luminance of the screen outside the circular window matched the mean luminance of the grating within the window. The size of the window was optimized for each cell. However, for some cells that were severely inhibited by an extended grating because of a strong suppressive surround, the size of the optimal window was approximately the same as, or even smaller than, one spatial period of the optimal grating. Orientation selectivity for such a small window will be biased toward low selectivity values. To avoid this situation, we adopted a criterion of at least two grating cycles within the diameter of the window. For those cells strongly inhibited by the surround, we ran experiments with a larger than optimal window to include at least two grating cycles, although this stimulus configuration was not optimal for the cell.

Receptive fields were located between 1 and $6^{\circ}$ from the fovea. A response criterion was imposed on the orientation tuning curves to exclude neurons that gave weak visual responses. Only cells that achieved spike rates of at least 5 spikes/sec above a threshold were included in the data set. The threshold was equal to the spontaneous rate of firing plus twice its SD. Spontaneous firing rates were measured with a uniform screen of the same mean luminance as that of the grating stimuli. In addition, only cells that were studied in some detail were included in our data set. This means that measurements of the spatial and temporal frequency tuning curves of the neuron, as well as a contrast response function, were available. Finally, cells for which we were unable to assign a laminar location were discarded. The total number of cells in the resulting database is $n=308$.

Modulation ratio. The modulation ratio $M=R(F 1) / R(F 0)$ for responses to optimal drifting sine gratings was measured as an estimator of the linearity of the response of a neuron (Enroth-Cugell and Robson, 1966; Maffei and Fiorentini, 1973; Skottun et al., 1991). $R(F 1)$ was calculated from the spike train as the amplitude of the best-fitting sinusoid at the modulation frequency of the drift, $F 1$. $R(F 0)$ was the mean spike rate during the drifting grating stimulus.

Circular variance and bandwidth. To study orientation selectivity across a large population of neurons, it is useful to have a single number for each orientation-tuning curve that measures the degree of selectivity of the neuron. We used two different measures in our analysis. Our first measure of selectivity was the circular variance of the response (Mardia, 1972; Batschelet, 1981; Levick and Thibos, 1982; Wörgötter and Eysel, 1987; Leventhal et al., 1995; Sato et al., 1996; Swindale, 1998). Circular variance is quite robust to noise in the data and provides a bounded "index" of orientation selectivity ranging from 0 to 1 (Mardia, 1972; Batschelet, 1981).

The circular variance was calculated from orientation tuning curves as follows. We measured the mean spike rates, $r_{k}$, in response to a grating drifting with angle $\theta_{k}$. The angles $\theta_{k}$ spanned the range from 0 to $360^{\circ}$ with equally spaced intervals. From these data, the circular variance of 
the responses in the orientation domain is defined as $V=1-|R|$, where $R$ is the resultant, calculated from the data as follows:

$$
R=\frac{\sum_{k} r_{k} e^{i 2 \theta_{k}}}{\sum_{k} r_{k}}
$$

where the angles are expressed in radians.

The circular variance averages the responses for the two directions of motion at each orientation. If there is no orientation tuning, such that the value of $r_{k}=C$ (a constant) for all $k$, then $V=1$. If an orientation-tuned neuron is so exceptionally selective that its responses $r_{k}$ are zero except for one nonzero response at its one preferred angle, then $V=0$. Thus, highly selective cells are mapped to values of $V$ close to 0 , and those with weak selectivity are mapped to values of $V$ close to 1 .

An equivalent, and perhaps more intuitive, description of the measurement performed by the circular variance is the following. We can fit a cosine function to the orientation tuning data, $r(\theta)=A+B \cos (2(\theta-$ $\left.\left.\theta_{\text {pref }}\right)\right)$. Here, the parameter $A$ represents the mean response of the cell across all orientations, $B$ is the amplitude of the modulation of the response with orientation, and $\theta_{\text {pref }}$ is the preferred orientation angle for the cell. The circular variance of the response is then $V=1-B / 2 A$. This number is one minus the "contrast," or relative modulation, of the cosine fit to the data.

The second measure of selectivity we used was the tuning curve half-bandwidth at $1 / \sqrt{2}$ height as has been used previously (Schiller et al., 1976). This measure of selectivity was calculated from the data as follows. The direction tuning data were first smoothed with a Hanning window filter (Rabiner and Gold, 1975), with a half-width at half-height of $13.5^{\circ}$. Then, the location of the peak of the tuning curve was determined. The orientation angles closest to the peak for which the response equals $1 / \sqrt{2}$ (or $70.7 \%$ ) of the peak response on either side of the curve were estimated. Bandwidth was defined as one-half of the difference between these two angles. If the tuning curve never went below response criterion, the bandwidth is defined as $180^{\circ}$. To abbreviate, we refer to the half-bandwidth at $1 / \sqrt{2}$ height simply as the bandwidth of the cell. The two measures, circular variance and bandwidth, provide different information about the shape of the tuning curve. Circular variance is a global measure that is influenced by all of the data points on the tuning curve. Bandwidth is a more local measure that depends on the shape of the curve around its peak and is not sensitive at all to the shape of the curve lying below $1 / \sqrt{2}$ of the peak response. Both measures can be considered reasonable definitions of "selectivity." However, as we show below, they do not always agree.

The selectivity measures were calculated based on the mean spike rate of the neurons during the response to a visual stimulus. For cells with a high modulation ratio, one could also define a similar measure with $r_{k}$ representing the first harmonic amplitude $(F 1)$ of the response. For cells with a high modulation ratio, we found that the values of circular variance based on $F 0$ and $F 1$ were very highly correlated ( $r=0.987$ ). Thus, the selection of the response measure as $F 1$ does not influence the results presented below.

We also decided not to subtract the spontaneous rate of the responses from the visually driven responses before the calculation of circular variance and bandwidth. Rather, the circular variance was calculated on the response $r_{k}$ defined as the mean spike rate during a stimulus presentation. This was done because we wanted a measure that represents how much the response of the cell is modulated with orientation and not the degree to which the response to an oriented pattern is different from spontaneous. It is also of interest to ask whether there is any relationship between tuning selectivity and the spontaneous rate of the neurons. We offer such an analysis in Results.

Finally, whenever orientation-tuning curves are plotted in the range from 0 to $180^{\circ}$, we averaged the responses for the two directions of drift at each orientation.

The data discussed in this paper are available for download at http:// manuelita.psych.ucla.edu/ dario/neurodata.htm.

\section{RESULTS}

\section{Circular variance and bandwidth in the $\mathbf{V} 1$ population}

There is a wide diversity of orientation selectivity in V1 cortex. Over the whole cell sample, there is a rather flat distribution over

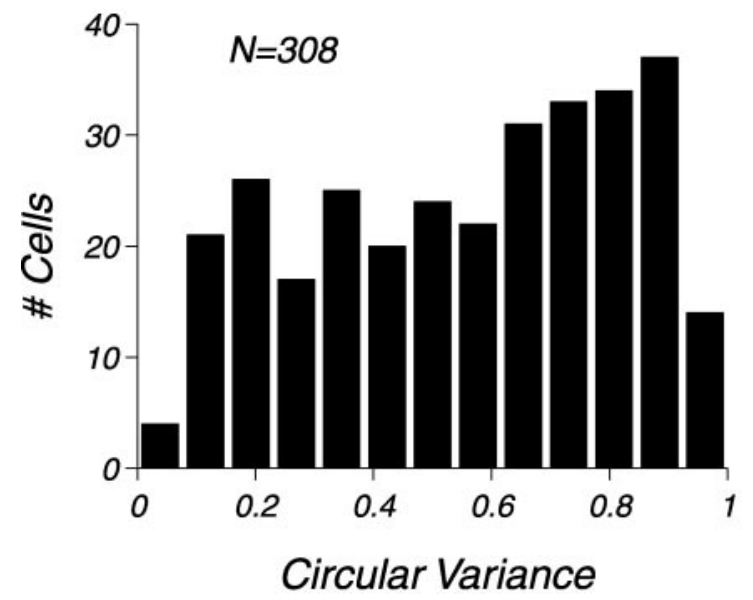

Figure 1. Distribution of circular variance for the V1 population. Circular variance is defined in Materials and Methods.

the entire circular variance range (Fig. 1). The median circular variance of this broad distribution is 0.61 , which is the circular variance of a not very selective neuron. However, there are many $\mathrm{V} 1$ cells with circular variance $<0.4$, indicating a relatively high degree of selectivity (see the individual tuning curves in Fig. 3).

Figure 2 illustrates the distribution of orientation bandwidth across the V1 population. The median bandwidth is $23.5^{\circ}$, and the distribution of bandwidth is skewed to higher values. These results are in good agreement with the previous findings about bandwidth in V1 by Schiller et al. (1976) and DeValois et al. (1982).

\section{Circular variance and bandwidth: direct comparison}

To understand better the relationship between the circular variance and bandwidth measures, we constructed a scatterplot of circular variance versus bandwidth for our population of V1 neurons (Fig. 3). Orientation bandwidths between 0 and $40^{\circ}$ are represented by most of the circular variance range, from 0 to 0.8 (Swindale, 1998). Cells with bandwidths larger than $40^{\circ}$ are mapped to values of circular variance between 0.8 and 1.0. However, often a single value of orientation bandwidth will be mapped to many different circular variance values, and it is interesting to understand why this occurs.

As we show in Appendix, one can derive a formula that relates circular variance to bandwidth for orientation tuning curves that approximate the shape of the most selective V1 tuning curves (a triangular-shaped tuning curve with zero response outside the tuning band). The formula is as follows:

$$
V=1-\sin ^{2}(B) / B^{2},
$$

where $V$ is circular variance, and $B / 2$ is half-bandwidth at halfheight, in units of radians. The prediction of the circular variance versus bandwidth from this equation constitutes a curve that runs approximately parallel to the bottom boundary of the cloud of data points in Figure 3 (see Appendix and Fig. 16). However, many neurons have orientation tuning curves with nonzero response at all orientations. For neurons with these less selective orientation tuning curves, Equation 1 relating circular variance to bandwidth does not predict the circular variance. In these cases, circular variance is larger than predicted from the simple formula because of additive contributions to circular variance from responses to angles far from the preferred orientation. One can derive a more general formula for the relationship between an orientation tuning curve and its circular variance (see Appendix). 


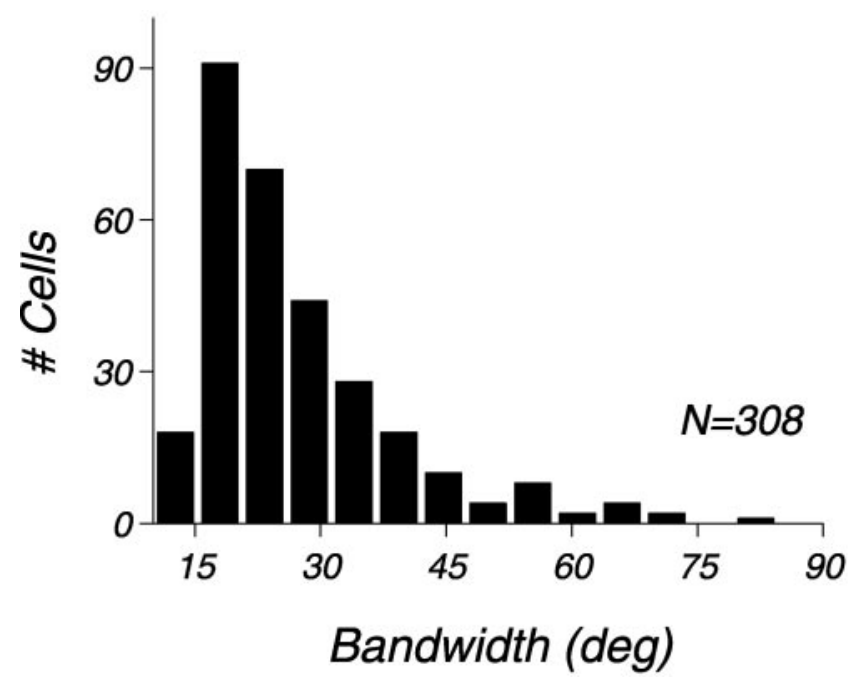

Figure 2. Distribution of half-bandwidth at $1 / \sqrt{2}$ height for the V1 population.

If a tuning curve is not zero outside the tuning band but instead has a baseline response $r_{0}$ and the peak response is $r_{0}+r_{p}$, then the relationship between circular variance, bandwidth $B$, and $c=$ $r_{0} / r_{p}$ is as follows:

$$
V=1-\Omega \sin ^{2}(B) \text { where } \Omega=1 /\left(B^{2}+2 \pi B c\right) .
$$

Some sample functions relating circular variance and bandwidth in this case are graphed in Figure 16 in Appendix, for different values of $c$, the ratio of wide angle to peak response.

To get an intuition for the differences between circular variance and bandwidth, it helps to inspect individual examples from Figure 3. In Figure 3, the pairs $a, b$ and $c, d$ are examples of cells with similar bandwidth but quite different circular variance. Examining the tuning curves, one sees that indeed the curves have similar shape around their peak. However, the responses near the orthogonal orientation are quite different. In both $a$ and $c$, orthogonal stimulation produces a response very close to zero, whereas in $b$ and $d$, orthogonal stimulation produces a significant response. This feature is picked up by the circular variance measure. Similarly, the pairs $d, f$ and $b, e$ are examples of cells with similar circular variance but quite different bandwidths. The cases in which the circular variance and bandwidth measures disagree illustrate how these two measures are indicating different aspects of orientation selectivity: bandwidth depends on the shape of the tuning curve around the peak, whereas circular variance weights responses at all orientations in its estimate of selectivity.

\section{Laminar distribution of circular variance}

The results on laminar distribution of orientation selectivity using the circular variance measure reveal the diversity and the laminar specialization of V1 cortex. Figure $4 a$ depicts a scatterplot of circular variance versus depth in the cortex. Figure $4 b$ shows curves that graph descriptive statistical measures of the population data. The middle (thick) curve represents a moving median of the circular variance data through the depth of the cortex using a window width of $100 \mu \mathrm{m}$. This curve was obtained by selecting, at each cortical depth, all of the data points from cells that were no more than $50 \mu \mathrm{m}$ above and below and then computing the median of their circular variance. Similarly, the curve with the thinner line to the left of the median curve represents the first quartile of the data, and the curve to the right of the median curve represents the third quartile.

Figure 4 suggests a revision of the classical view that nonoriented receptive fields are principally found in layer $4 \mathrm{C}$ and the cytochrome oxidase-rich blobs in layer $2 / 3$, whereas only highly selective neurons are found outside these regions. In fact, there is a broad distribution of circular variance in all layers of V1. It can be seen that neurons with large circular variance (low selectivity) are present in all layers. The behavior of the third quartile curve demonstrates that at least $25 \%$ of the cells in all layers have a circular variance greater than 0.65 . Cells with this value of circular variance usually respond at all orientations. A trend in the data suggests that weakly selective neurons are predominant in layers 3B, 4C, and 5. A multiple comparison test between pairs of layers, however, reveals that these differences are not significant given the current amount of data (pairwise Wilcoxon test with Bonferroni's correction; $p>0.1$ in all cases).

\section{Laminar distribution of orientation bandwidth}

There also is a range of orientation bandwidth throughout all layers of V1 as illustrated in the laminar scatterplot and average statistical measures in Figure 5, $a$ and $b$. Although Figure 3 illustrates that bandwidth and circular variance of a single cell need not agree in their assessment of selectivity, there is some concordance in the laminar patterns for these two different measures. For instance, there is a larger fraction of broad-bandwidth cells in layer $4 \mathrm{C}$ and layer $3 \mathrm{~B}$ than in other layers. This is particularly evident in the third quartile statistic. The third quartile of bandwidth approximately parallels the variation of median circular variance with cortical depth, but median bandwidth is approximately constant through the depth of the cortex.

\section{Comparison of circular variance and bandwidth with another measure of selectivity}

It is interesting to compare the orientation selectivity measures we are using in this paper with different selectivity measures used by others. One such measure is the response at the orthogonal orientation divided by the response at the preferred orientation. This measure was used by Gegenfurtner et al. (1996) in their study of orientation selectivity in V2 neurons. It resembles a related selectivity index used by Zhou et al. (2000) in a study of V1 and V2 neurons. A graph of a scatterplot of orthogonal/ preferred ratio versus circular variance is shown in Figure $6 a$. For circular variance $<0.5$, the orthogonal/preferred ratio is very close to zero. For values of circular variance $>0.5$, the orthogonal/preferred ratio is approximately proportional to the circular variance. Clearly, these two global measures of orientation selectivity are strongly correlated, but circular variance distinguishes between tuning curves that all have zero orthogonal/preferred ratio. Another way of stating the relationship is to write that low $(<0.5)$ values of circular variance can only occur when the orthogonal/preferred ratio is very close to, or equals, zero. This empirical observation is supported by the analysis of tuning curves and the relationship between off-peak responses and circular variance offered in Appendix.

The relationship between bandwidth and orthogonal/preferred ratio is shown in Figure $6 b$. In that plot, there is much less covariation of the two measures than between circular variance and orthogonal/preferred ratio shown in Figure $6 a$. The implication of Figure 6 is that the neural factors that cause low values of orthogonal/preferred ratio also lead to low values of circular 


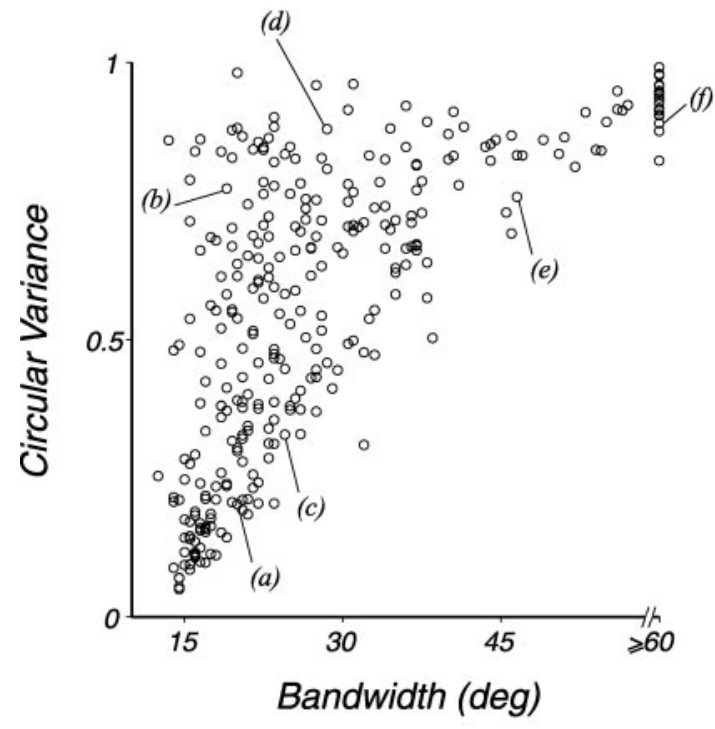

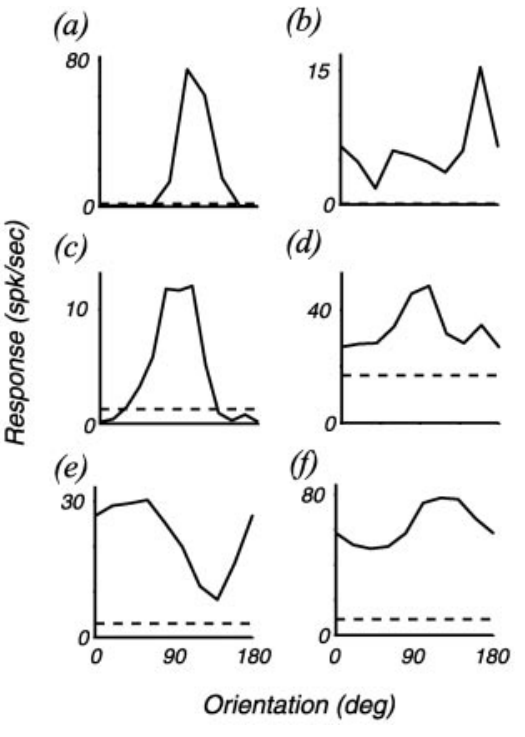

Figure 3. Relationship between orientation bandwidth and circular variance. Scatterplot of orientation bandwidth and circular variance for all cells in the measured V1 population. Cells with bandwidth values larger than $60^{\circ}$ are plotted at $60^{\circ}$ to make better use of the range of the $x$-axis. $a-f$, Examples of individual tuning curves in different locations of the scatterplot. The $x$-axis represents stimulus orientation, and its scale is the same for all graphs, from 0 to $180^{\circ}$, as indicated in the bottom plots. The $y$-axis is the response of the cell in spikes per second. The lower limit on the $y$-scale is zero for all graphs, and the upper limit is indicated in each case. The dashed line represents the spontaneous rate of firing. In those examples in which the line is not visible, it means that the spontaneous rate was zero. (a)

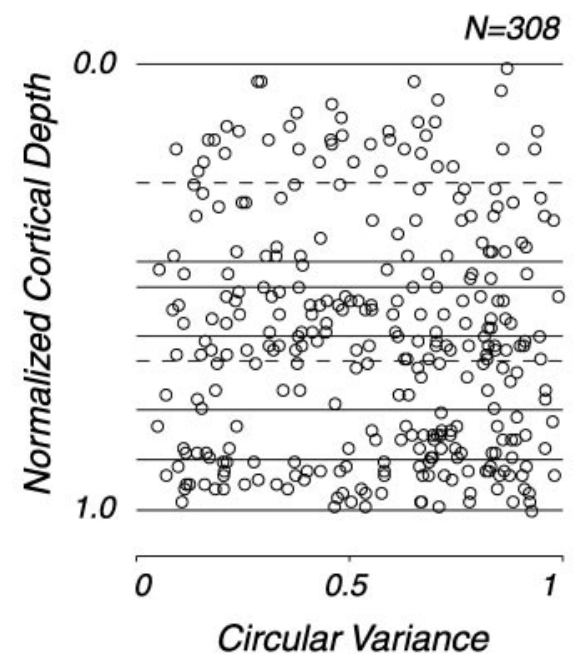

(b)

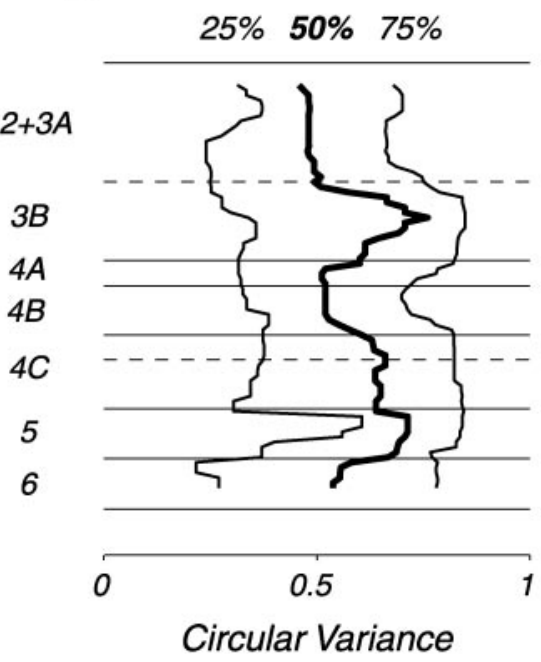

Figure 4. $a$, Plot of circular variance against relative cortical depth. $b$, Statistical summary of the scatterplot data in $a$. The middle curve drawn with a thicker line represents the median circular variance at different cortical depths. A window size of $100 \mu \mathrm{m}$, centered at each location, was used. The thinner curves to the left and right represent the first and third quartiles of the distribution. Horizontal lines represent the laminar boundaries. Details about the histological reconstruction can be found in Hawken et al. (1988). variance. However, the neural factors that influence bandwidth and orthogonal/preferred ratio are not so closely related. Because of the indication that low response far from preferred orientation is crucial for low circular variance, we next considered the effect of the spontaneous activity on circular variance and bandwidth.

\section{Population and laminar distributions of spontaneous activity}

Orientation selectivity could be related to spontaneous activity if the threshold level and the excitatory/inhibitory balance, which both influence spontaneous activity, also have a large influence on orientation tuning. Figure 7 illustrates the laminar dependence of spontaneous firing rate as a scatterplot of rate with cortical depth. It is evident that many cells throughout V1 have very low or zero spontaneous activity.

\section{Relationship between selectivity and spontaneous activity}

A natural question to ask is whether or not there is a relationship between the spontaneous rate of firing of a cell and its orientation selectivity. The relationship between orientation selectivity and spontaneous activity in our population is illustrated in Figure 8. The scatterplot in Figure $8 a$ is for spontaneous firing rate versus circular variance, whereas the scatterplot in Figure $8 b$ is for spontaneous firing rate versus bandwidth. It can be seen that cells that have low circular variance $(<0.4)$ all have very low spontaneous rates. Cells with high circular variance can have either high or low spontaneous rates. Thus, although there is a correlation between circular variance and the spontaneous rate of the cells, the distribution of circular variance cannot be explained entirely in terms of the factors that control spontaneous activity. Indeed, if we omitted from consideration all V1 cells that have a nonzero spontaneous firing rate, there would still be a very large amount of diversity in circular variance. This is evident from Figure $8 a$. Bandwidth also has a weak correlation with spontaneous activity. Figure $8 b$ shows that cells with the lowest bandwidth $\left(<20^{\circ}\right)$ tend to have very low spontaneous rates.

\section{Circular variance, spontaneous activity, and the orthogonal response}

Additional analysis of the population data reveals that other factors, besides those that govern the spontaneous firing rate, determine orientation selectivity. Figure 9 illustrates this important point. Here we plot spontaneous firing rate versus the firing rate at the orientation orthogonal to the preferred (often the 
(a)

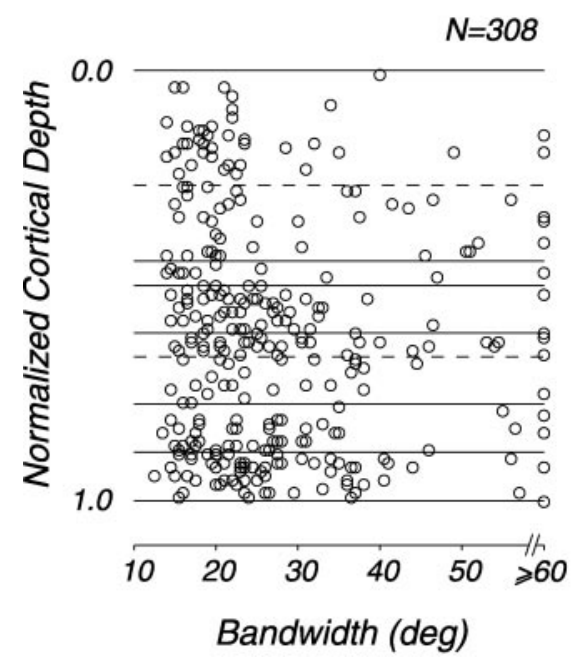

(a)

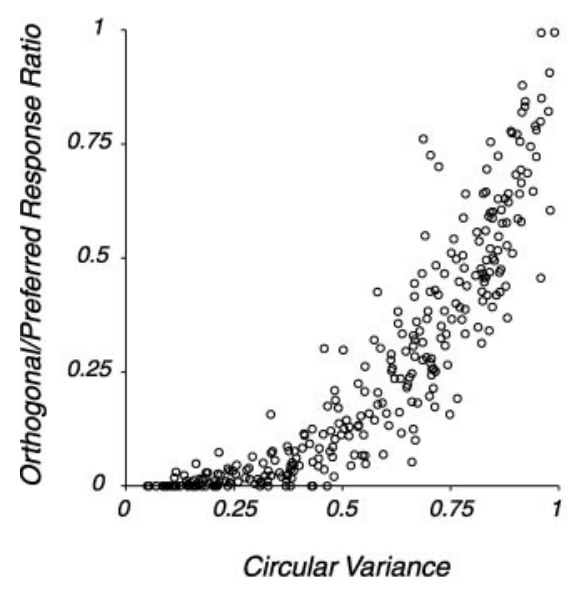

(b)

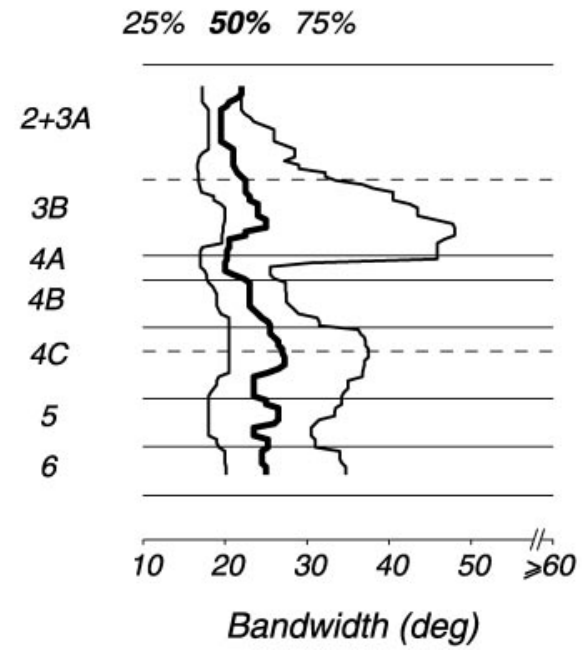

(b)

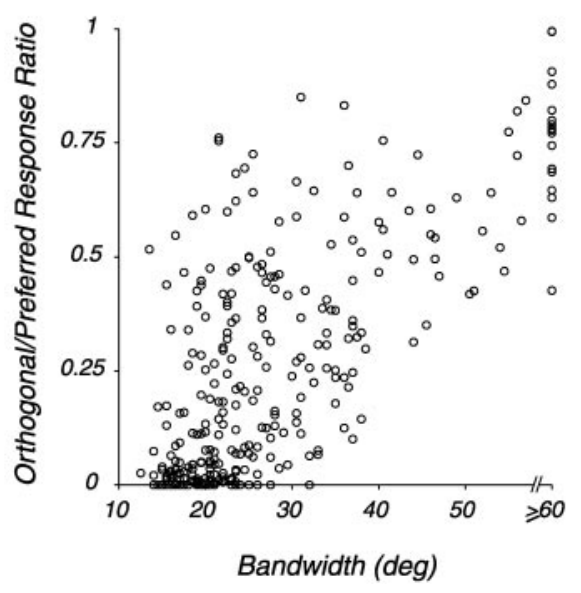

Figure 6. $a$, Relationship between circular variance and orthogonal/preferred orientation ratio. $b$, Relationship between orientation bandwidth (half-width at $1 / \sqrt{2}$ height) and the orthogonal/ preferred orientation ratio.

Figure 5. $a$, Plot of bandwidth against relative cortical depth. Cells with bandwidth values use of the range of the $x$-axis $b$, Statistical summary of the scatterplot data in $a$. The middle curve drawn with a thicker line represents depths. A window size of $100 \mu \mathrm{m}$, centered at each location, was used. The thinner curves to the left and right represent the first and third ment and relative depth are as described in Figure 4.

lowest visually driven firing rate on the orientation tuning curve). As before, cells with zero spontaneous rate are plotted with $y$-coordinate 0.1 . Cells with zero orthogonal response are plotted with an $x$-coordinate of 0.1 . The size of the data point represents the circular variance of the orientation tuning curve of the cell, as depicted by the scale to the right of the scatterplot. The larger the size of the data point, the better tuned the cell is.

For a group of neurons (62 of 308; 20.1\%), both the spontaneous and the orthogonal firing rates were zero, and these are all plotted at the bottom left corner of the graph. Excluding this group of cells, we can say that points above the diagonal represent cases in which the response at the orthogonal was lower than the spontaneous rate of the cell, thereby indicating suppression at the orthogonal orientation. It is worth noting that there are many such cells in our V1 sample (146 of 246; 59.4\%) (Ringach et al., 2002). Data points below the diagonal represent neurons for which the response at the orthogonal was larger than the spontaneous response ( 100 of $246 ; 40.6 \%$ ). The tuning curves of these neurons, especially those well below the diagonal, appear to be riding on top of a "pedestal," which suggests the presence of an unoriented component, as illustrated by the examples in Figure $3, b, d$, and $f$. Cells that are along or near the diagonal are cases for which the spontaneous and the response at the orthogonal were very similar one to the other, and circular variance tends to increase as one moves up the diagonal.

There are a number of conclusions that one can infer from this graph. First, it is apparent that cells with low circular variance are located above the main diagonal, confirming the suggested role of inhibition in the generation of high orientation selectivity (Ringach et al., 2002). Second, cells that lie on the vertical axis, where orthogonal $=0$, are almost all highly orientation selective, with low circular variance, but span a large range of spontaneous values. In contrast, one can observe many neurons below the main diagonal that have very low $(<1 \mathrm{spike/sec})$ spontaneous rates but are not very orientation selective. This last finding observed in Figure 9 is also evident in Figure $8 a$ (there are many cells in the top left). These observations, in agreement with the data in Figure $6 a$, suggest that the neuronal mechanisms that control the response at the orthogonal are more critical than the level of spontaneous activity in determining the circular variance of the neuron.

\section{Modulation ratio}

V1 neurons differ greatly in their temporal patterns of response to drifting gratings (DeValois et al., 1982; Skottun et al., 1991) (for results on cat area 17 neurons, see Maffei and Fiorentini, 1973; Movshon et al., 1978). At one extreme, the spike rate of the 
(a)

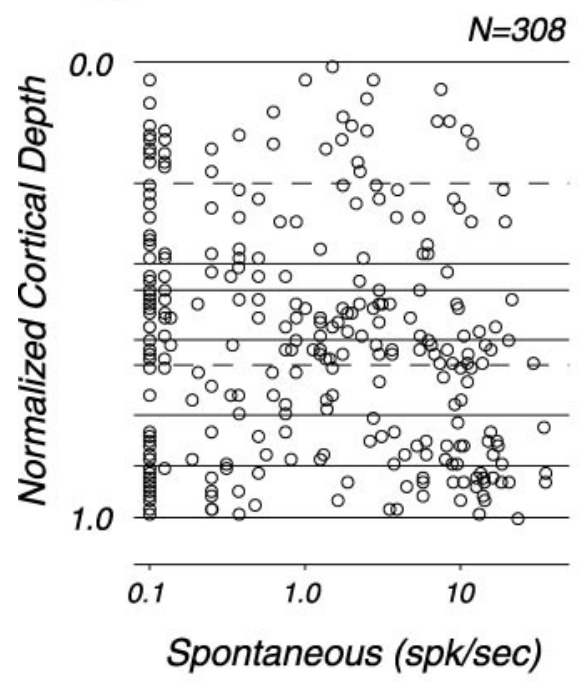

(a)

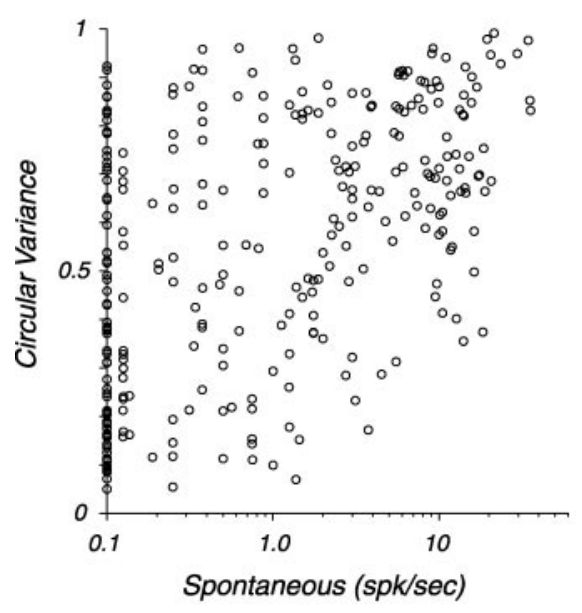

(b)

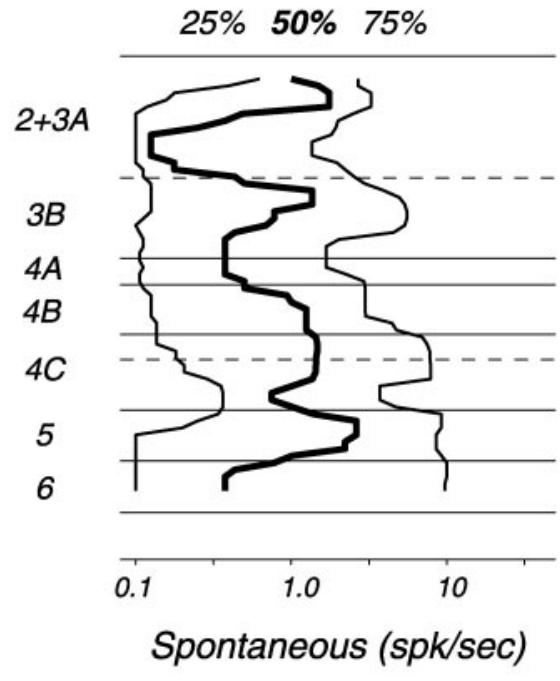

(b)

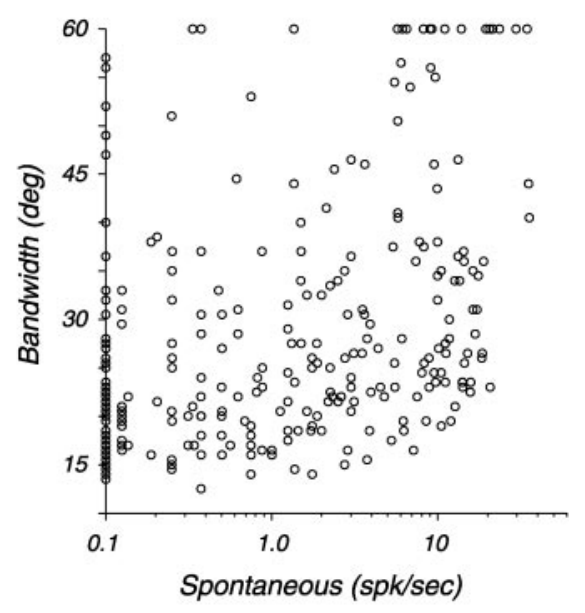

Figure 7. $a$, Plot of spontaneous firing rate against relative cortical depth. Cells with zero spontaneous rate are plotted at 0.1. The layer assignment and relative depth are as described in Figure 4. $b$, Statistical summary of the scatterplot data in $a$. The middle curve drawn with a thicker line represents the median spontaneous rate at different cortical depths. A window size of $100 \mu \mathrm{m}$, centered at each location, was used. The thinner curves to the left and right represent the first and third quartiles of the distribution.

Figure 8. Relationship between orientation selectivity and spontaneous firing rate. $a$, Circular variance. $b$, Bandwidth $(1 / \sqrt{2}$ height $)$. Cells with zero spontaneous rate are plotted at 0.1 .

neuron is modulated strongly at the rate of drift, and such neurons have been classified previously as simple cells (Skottun et al., 1991). At the other extreme, some neurons simply elevate their rate of spike discharge when presented with a drifting stimulus, and the rate is not modulated with the drift rate of bars crossing the receptive field of the neuron. Such neurons have been classified previously as complex cells (Skottun et al., 1991). One can quantify these patterns of response by calculating the modulation ratio $M=R(F 1) / R(F 0)$, where $R(F 1)$ is the amplitude of the best-fitting Fourier component at the drift rate, and $R(F 0)$ is the mean spike rate during stimulation.

In the distribution of modulation ratio for our sample of 308 cells (Fig. 10), there is a clear bimodality of the modulation ratio distribution similar to the bimodality used by Skottun et al. (1991) to classify cells as simple and complex. However, recently, Mechler and Ringach (2002) have shown that the bimodality of the modulation ratio distribution based on impulse rate does not necessarily imply that there is an underlying bimodal distribution of linear-nonlinear summation in the membrane potential. It is therefore still an open question whether or not there are two distinct classes of neurons based on the linearity or nonlinearity of spatial summation as measured by the modulation ratio. Nevertheless, large differences in modulation ratio between different neurons could be functionally significant. Theoretical work has indicated that the properties of cells with a high modulation ratio (Wielaard et al., 2001) and cells with very low modulation ratio (Chance et al., 1999) could be related to different patterns of intracortical functional connectivity. Specifically, to have a high modulation ratio, neurons in the model by Wielaard et al. (2001) needed to have the balance between synaptic excitation and inhibition tilted toward more inhibition to cancel out the nonlinear signals that would cause the modulation ratio to be lower. Conversely, to obtain complex cell-like (low modulation ratio) behavior in their model, Chance et al. (1999) assigned recurrent excitation greater strength than recurrent inhibition. There have been a number of different theoretical ideas for how to cause intracortical sharpening of orientation selectivity by means of cortico-cortical inhibition (Sillito, 1977; Bonds, 1989; McLaughlin et al., 2000) or by means of recurrent cortico-cortical excitation (Ben-Yishai et al., 1995; Douglas et al., 1995; Somers et al., 1995; Carandini and Ringach, 1997). Therefore, we studied the relationship between modulation ratio and circular variance with the idea that the results might provide clues about which corticocortical interactions might be playing a role in shaping orientation selectivity. 


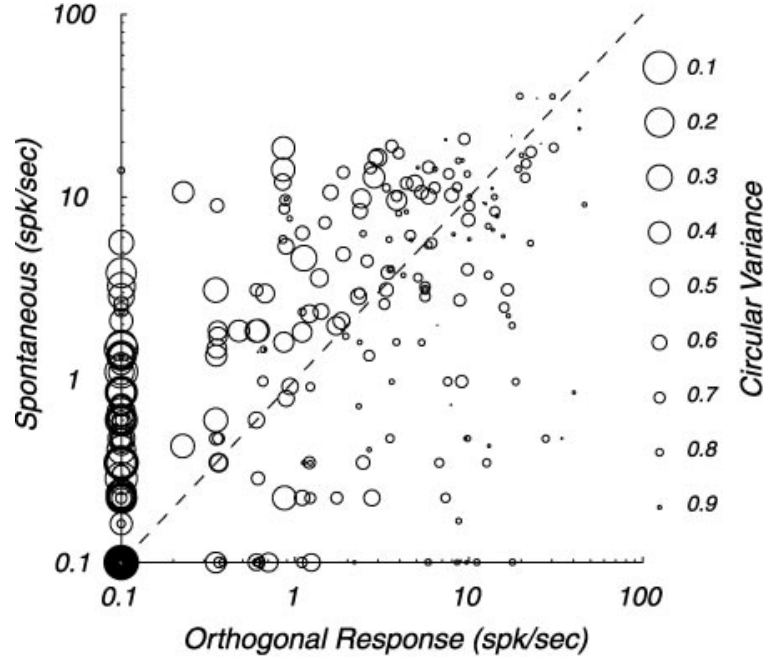

Figure 9. Dependence of circular variance as a function of spontaneous rate and the response at the orthogonal. The graph shows a scatterplot of the response at the orthogonal orientation versus the spontaneous rate of the neuron. The size of each data point corresponds to the circular variance of the tuning curve of the cell as illustrated by the scale on the right. Cells with a zero spontaneous rate are plotted with a $y$-coordinate of 0.1 . Cells with a zero orthogonal response are plotted with an $x$-coordinate of 0.1 .

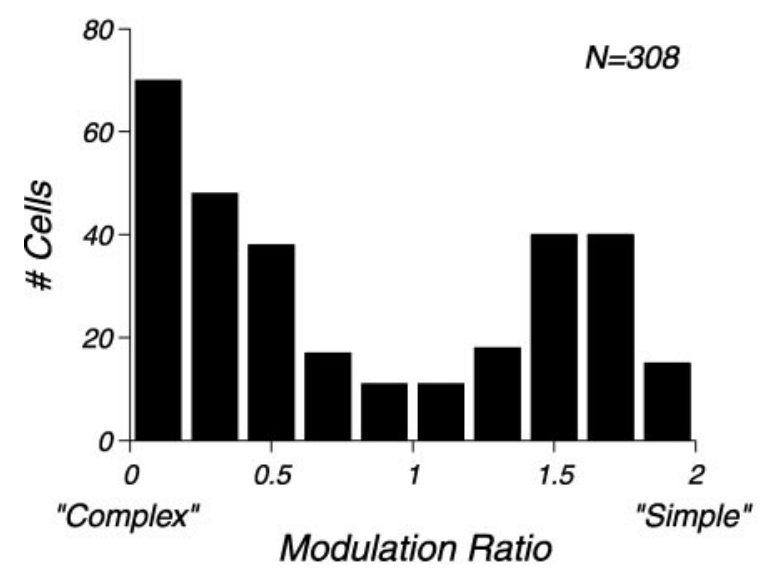

Figure 10. The distribution of modulation ratio. The modulation ratio is the amplitude of first harmonic $R(F 1)$ divided by the mean spike rate $R(F 0)$ for an optimal achromatic drifting sinusoidal grating stimulus. High values of $R(F 1) / R(F 0)$ indicate that the cells are modulated by spatial pattern in the visual image. Low values of $R(F 1) / R(F 0)$ signify that such cells are excited, but their spike rate is not modulated up and down by the passage of the bars of a drifting grating.

\section{Laminar distribution of modulation ratio}

In exploring the possible relationship between modulation ratio and orientation selectivity, we thought it was necessary to establish the laminar pattern of modulation ratio. This would enable a comparison with the laminar patterns of circular variance and bandwidth (Figs. 4, 5). Data are shown in Figure 11. The median of the modulation ratio is drawn overlaid on the scatterplot of modulation ratio versus cortical depth. The median of the modulation ratio peaks in the input layers $4 \mathrm{C} \alpha$ and $\beta$. All other layers in the cortex have a lower median modulation ratio, with layers $3 \mathrm{~B}, 4 \mathrm{~B}$, and 5 having the lowest medians. Because the laminar dependencies of circular variance and bandwidth do not follow this pattern, we sought to establish whether or not there was a

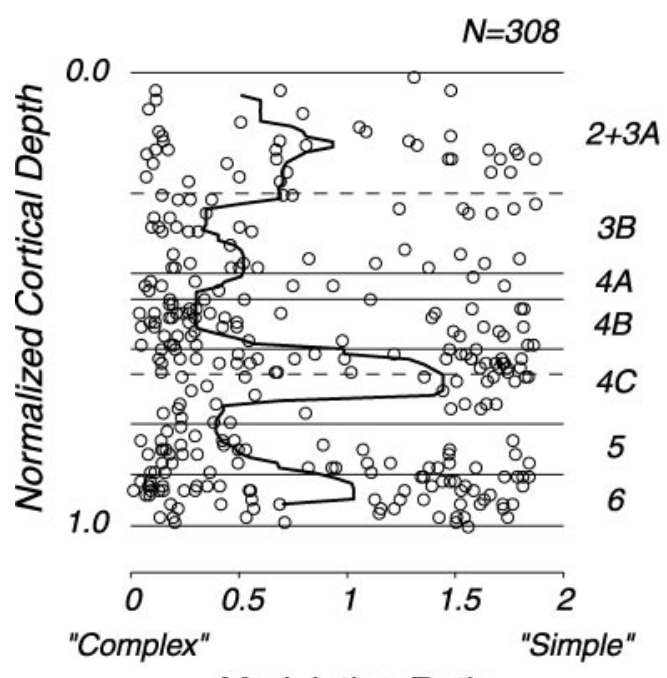

Modulation Ratio

Figure 11. Plot of modulation ratio against relative cortical depth. The continuous thin line gives the running median of the data using a window size of $100 \mu \mathrm{m}$, centered at each location. The layer assignment and relative depth are as described in Figure 4.

correlation of the orientation selectivity measures with modulation ratio on a cell-by-cell basis, as follows.

\section{Relationship between modulation ratio and circular variance}

There is an interesting relationship between modulation ratio and circular variance (Fig. 12a). Figure $12 b$ shows a color density plot of the smoothed joint distribution of circular variance and modulation ratio that shows that there is clustering into distinct groups. In this plot, the density of neurons has been smoothed with a two-dimensional Gaussian, with $\sigma_{\mathrm{x}}=0.2$ (modulation ratio) and $\sigma_{\mathrm{y}}=0.1$ (circular variance). The color in the graph encodes the density of neurons per bin. Cells with low modulation ratio form a large cluster with high circular variance at the top left corner of the plot. The cells of high modulation ratio form two separate clusters toward the right of the graph, at higher and lower circular variance. The existence of the cluster at high circular variance and low modulation ratio suggests that the network or biophysical factors that cause a cell to have a low modulation ratio also may cause it to be less selective for orientation. To explore this further, we also studied the covariation between modulation ratio and bandwidth.

\section{Relationship between modulation ratio and bandwidth}

The modulation ratio appears to be less closely related to bandwidth than to circular variance. A scatterplot and color density of the smoothed joint distribution of bandwidth and modulation ratio are displayed in Figure 13. In Figure 13b, the density of neurons has been smoothed with a two-dimensional Gaussian, with $\sigma_{\mathrm{x}}=0.2$ (modulation ratio) and $\sigma_{\mathrm{y}}=2.5^{\circ}$ (bandwidth). The two clusters of neurons in the (bandwidth, modulation ratio) plane are located at approximately the same values of bandwidth. The different covariations of circular variance and bandwidth with modulation ratio is yet more evidence of the dissociation between the mechanisms that produce orientation bandwidth and those that determine circular variance. 
(a)

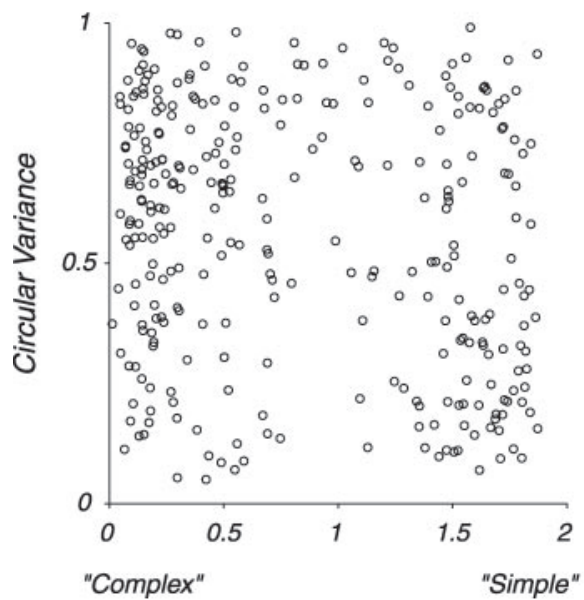

Modulation Ratio (b)

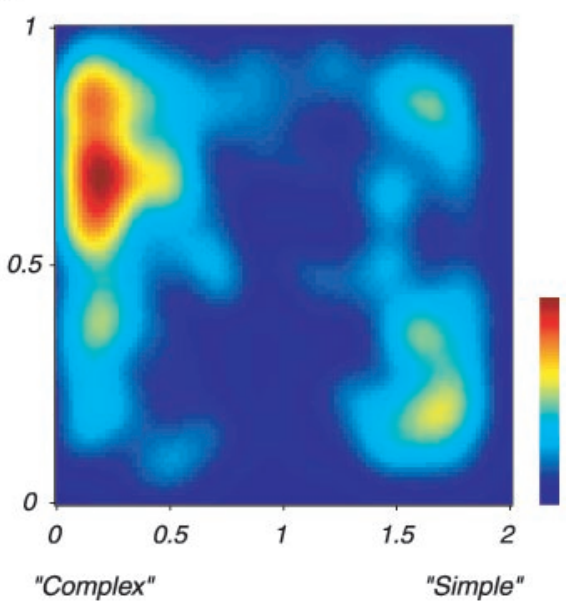

Modulation Ratio

(b)

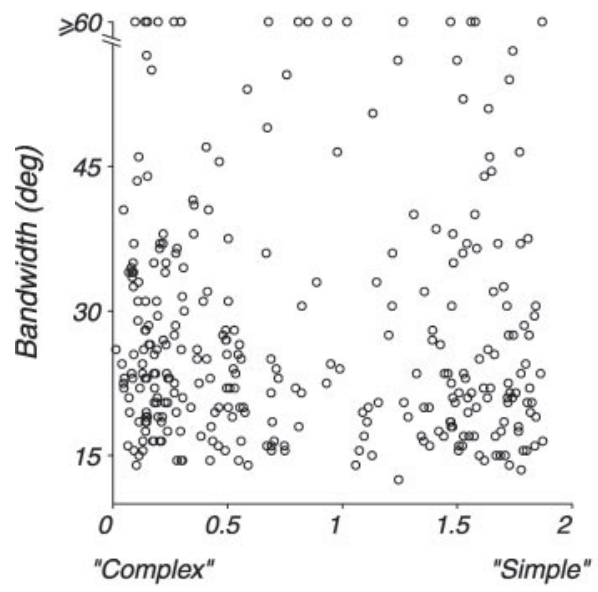

Modulation Ratio

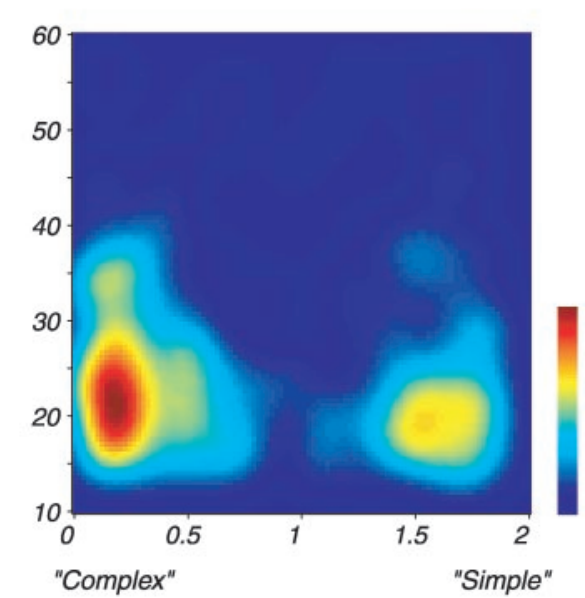

Modulation Ratio
Figure 12. $a$, Plot of circular variance against modulation ratio. $b$, A color-coded density of smoothed joint distribution of circular variance versus modulation ratio. The color scale represents the relative density of neurons in the distribution and ranges from 0 (blue) to 1 (red).
Figure 13. a, Plot of orientation bandwidth (1/ $\sqrt{2}$ height) against modulation ratio. $b, \mathrm{~A}$ color-coded density of smoothed joint distribution of orientation bandwidth versus modulation ratio. The color scale represents the relative density of neurons in the distribution and ranges from 0 (blue) to 1 (red).

\section{Relationship between modulation ratio and spontaneous activity}

The average spontaneous activity in low modulation ratio cells is significantly larger than in high modulation ratio cells (Wilcoxon rank sum test; $p<10^{-8}$ ). This difference is worth noting because it may be related, at the level of cellular mechanisms, to the elevated circular variance of the low modulation ratio cells relative to high modulation ratio cells that was shown in Figure 12. The joint distribution of modulation ratio and spontaneous activity (Fig. 14) reveals the very low spontaneous activity of the high modulation ratio cells. There is also a peak near zero spontaneous activity for the low ratio cells but also a significant probability of a neuron with a low modulation ratio to have a spontaneous rate above zero. Thus, circular variance, modulation ratio, and spontaneous activity all seem to be correlated to some extent. Bandwidth seems less closely related to any of these measures of neuronal activity.

\section{DISCUSSION}

\section{Diversity}

The most striking result of this study is the wide diversity of orientation selectivity in the population of macaque V1 neurons.
This diversity is not simply a consequence of differences in selectivity between cells in different cortical layers because the data indicate that it is present in all layers. The diversity is particularly evident in the circular variance data. Examination of the tuning curves in Figure 3 suggests that multiple factors may be causing the wide spread in circular variance. It appears that a V1 tuning curve, response versus orientation angle, usually has a central "core" tuning band of orientations, and many show a wide plateau of responses across all orientations. Circular variance depends on the width of the core tuning but also on the relative height of the plateau response compared with the response at the preferred orientation. In Appendix (Figs. 15, 16), we show that this is the case for model tuning curves for which the relationship between bandwidth and circular variance can be calculated analytically. In the analysis, the two factors that affect circular variance (bandwidth and peak-plateau response) are independent, in principle. This focuses attention on the factors that could cause variation in the relative height of the plateau compared with the peak response and also on what factors could cause variation in orientation bandwidth.

The presence of a plateau in orientation tuning curves arises naturally in feedforward models of V1 receptive fields (for re- 


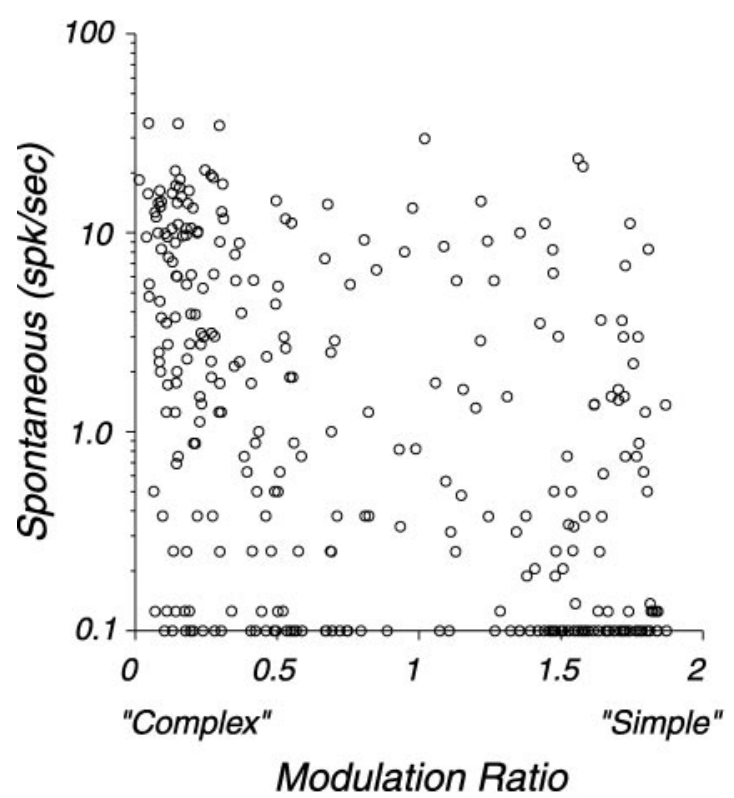

Figure 14. Plot of spontaneous firing rate against modulation ratio. Cells with zero spontaneous rate are plotted at 0.1 .

view, see Sompolinsky and Shapley, 1997; Ferster and Miller, 2000). Usually, theoretical models of orientation tuning are designed to remove the plateau, usually by means of cortical inhibition (Troyer et al., 1998; McLaughlin et al., 2000). This is because most illustrations of orientation tuning curves show curves without plateaus (Sclar and Freeman, 1982; Anderson et al., 2000). Nevertheless, plateaus are often observed, well above the level of the spontaneous activity (Fig. $3 c-f$ ). Although this phenomenon could simply be a trace of nontuned feedforward input from the LGN, it is also possible that it could be generated within the cortex by excitatory convergence onto a cortical cell from other, highly tuned, cortical cells that have a wide range of preferred orientations. The relative height of the plateau compared with the peak response is likely to depend on the relative strength of cortical excitation and inhibition. Thus, diversity in relative heights of plateau and peak, and thus in circular variance, are likely related to the balance of excitation and inhibition that is important for cortical function.

The factors that control bandwidth are likely to be different from those that determine circular variance. The aspect ratio of the feedforward LGN input, as well as the number of receptive field subregions, are major factors that affect bandwidth (Hubel and Wiesel, 1962; Jones and Palmer, 1987; Ferster, 1988). Quantitative measurements of the shapes of receptive fields, by means of reverse correlation techniques, are available and indicate that there may be enough variation in aspect ratio and number of subregions to account for the range of bandwidths observed (Jones and Palmer, 1987; Ringach et al., 2002). On the other hand, the direct (excitatory) feedforward input from the LGN does not provide the suppressive component that would be required to suppress the responses at orientations far from the peak that is needed to account for cells with both narrow bandwidth and low values of circular variance. Such suppression is evident in Figure 9 and in reverse correlation experiments we reported recently (Ringach et al., 2002). It is also possible that corticocortical suppression could also contribute to narrowing the bandwidth in highly selective neurons.

\section{Modulation ratio and orientation selectivity}

Next we consider the finding that modulation ratio and circular variance are correlated. One possible explanation for this correlation is that low ratio cells are generated by feedforward convergence from the high ratio cells (cf. Hubel and Wiesel, 1962). Massive convergence from neurons with a range of orientation preferences and also spatial phase preferences (receptive field positions) could account for the low modulation ratio and also offer an explanation for poor orientation selectivity. Another possibility is suggested by a recent model for complex cells, which hypothesizes that complex cells receive a large amount of recurrent excitation from lateral cortico-cortical connections (Chance et al., 1999). Such excitation from populations of cells with different orientation preferences could cause a reduced degree of selectivity in complex cells that comprise a large fraction of the neurons with a low modulation ratio. The recurrent excitation explanation might also explain why low ratio cells have, on average, higher spontaneous rates and higher circular variance than cells with a high modulation ratio. These would be a consequence of the greater amount of cortico-cortical excitation in these neurons. Both the feedforward and recurrent excitation explanations account for weak orientation selectivity by pooling of excitatory inputs from neurons with different preferences. Although this explanation accounts for the main body of the low ratio population, there are some highly selective low modulation ratio cells discussed below.

One needs also to account for the low circular variance among the group of neurons with a high modulation ratio. The cortical neurons with the highest modulation indices have traditionally been called simple cells. In a modeling study, Wielaard et al. (2001) proposed that simple cells must be "overinhibited." That is, to achieve a high modulation ratio, the cortex must generate strong inhibition (relative to net excitation from LGN and other cortical cells) to cancel out the "nonlinear" LGN and corticocortical excitation. Such strong inhibition could also be a mechanism for reduction of circular variance (by suppressing the plateau responses discussed above), as suggested by Troyer et al. (1998) and McLaughlin et al. (2000). This hypothesis is consistent with our own results on orientation dynamics in which sharp selectivity for orientation was associated with signs of suppressive interactions (Ringach et al., 1997, 2002). The hypothesis that cells with a high modulation ratio receive more intracortical inhibition is also consistent with the overall lower spontaneous firing rate of the high ratio cells, as illustrated in Figure 14.

It is possible that the small population of highly selective cells with low modulation ratio also get their high selectivity from greater amounts of inhibition. The reason for this conjecture is that all the neurons with low circular variance have near-zero spontaneous rates, as shown in Figure 8. However, some of these neurons are the low modulation ratio neurons, and so these must comprise a subpopulation of low modulation ratio cells with low circular variance and zero spontaneous rate. Perhaps this subgroup has low spontaneous and low circular variance because of strong cortico-cortical inhibition but low modulation ratio because of strong recurrent cortico-cortical excitation.

\section{Laminar patterns}

We found a significant diversity in orientation selectivity across all V1 layers. The circular variance distributions are broad. Cells with high selectivity and cells with low selectivity can be found in all cortical layers. These results suggest that orientation selectivity in macaque V1 is caused by mechanisms that affect responses 
in the input layers, as well as in the output layers. The previous results by Schiller et al. (1976) about bandwidth of cells in different layers are consistent with our results, but they may not have been interpreted as indicating quite as much diversity because of the compressive nature of the bandwidth measure.

\section{Layer 3B}

One salient new aspect of the data in Figures 4 and 5 is the cluster of broadly tuned cells in layer $3 \mathrm{~B}$ in the bottom one-third (approximately) of layer $2 / 3$. There are also some highly selective cells found in layer $3 \mathrm{~B}$, but they are found relatively less frequently than in other layers. Layer $4 \mathrm{C} \beta$ and the K-layers of the LGN both send substantial projections to layer 3B (Fitzpatrick et al., 1985; Lund, 1988; Hendry and Yoshioka, 1994; Ding and Casagrande, 1997, 1998). Previously, Blasdel and Fitzpatrick (1984) reported results on macaque V1 that agree with our findings. They wrote that a sequence of nonoriented units was observed just before entering layer $4 \mathrm{~A}$, followed by a responsive group of orientation tuned cells (probably layers 4A, 4B, and the top of $4 \mathrm{C} \alpha$ ) and then followed by a sequence of unoriented cells (layer $4 \mathrm{C} \alpha$ or $4 \mathrm{C} \beta$ ). Our results are the first quantitative, objective measurements of this subdivision of layer 3 .

\section{Comparison with results in other species}

The results presented in this paper may seem to indicate that orientation selectivity in macaque V1 is very different from that seen in primary visual cortex of cat and other mammalian species. This is because the diversity of selectivity, and the mean selectivity, as measured by mean circular variance of 0.60 , might be interpreted as indicating less selectivity in macaque than in other species. However, examination of the data available seems to indicate that our findings in macaque V1 are similar to what has been found in cat and ferret primary visual cortex. Recently, Dragoi et al. (2001) reported that the mean orientation selectivity index (OSI) of a population of 248 neurons they studied in cat V1 was 0.3 . OSI is $1-$ circular variance, so the mean circular variance they reported for their cat data was 0.7 , somewhat higher (less selective) than for our monkey data. Calculating the mean circular variance from unpublished data on a population of 350 neurons from cat V1 from C. M. Gray, P. E. Maldonado, and T. Bonhoffer (personal communication) yields a mean circular variance of $\sim 0.55$, also approximately in agreement with the macaque data reported here. Analogous measurements on a population of neurons from adult ferret V1 by Chapman and Stryker (1993) were approximately in agreement with our macaque data, with mean OSI of 0.4 (circular variance of 0.6 ). The ferret data differed from our macaque results in that Chapman and Stryker found that the mean OSI in the input layer 4 was markedly lower (OSI of 0.2 ; circular variance of 0.8 ) than in other layers of ferret cortex. It would be desirable if there were more data on the distribution of circular variance in other species, but the available evidence indicates that orientation selectivity in macaque V1 is similar to that observed in other mammalian species.

\section{APPENDIX}

One can derive a formula that relates circular variance to bandwidth for orientation tuning curves of different shapes. For tuning curves that approximate the shape of the most selective V1 tuning curves, we will use a triangular-shaped tuning curve with zero response outside the tuning band (this is equivalent to the tuning curve in Fig. 15 with $r_{0}=0$ ). This is an approximation that is not intended to be precise but does enable us to calculate the relationship between circular variance and bandwidth analytically. The aim is to use this approximation to get insight into the relationship between the two measures of orientation selectivity and not to fit the data precisely.

Without loss of generality, we can consider the triangular orientation tuning curve to be centered at approximately $\theta=0^{\circ}$. Then such a tuning curve can be described by the equation $r(\theta)=$ $r_{p}(1-|\theta| / B)$, for $|\theta|<B$, and $r(\theta)=0$ otherwise. The response intercept with the orientation axis $B$ is related to the bandwidth; it is twice the half-bandwidth at half-height, or $(1-1 / \sqrt{2})^{-1}$ $(\sim 3.4 \times)$ times the half bandwidth at $1 / \sqrt{2}$ height as we have used in this paper.

Recall that the circular variance of the responses in the orientation domain is defined as $V=1-|R|$, where $R$ is the resultant, calculated from the data as follows:

$$
R=\frac{\sum_{k} r_{k} e^{i 2 \theta_{k}}}{\sum_{k} r_{k}},
$$

For the continuous triangular tuning curve, the summation is replaced with an integral, and the result is as follows:

$$
V=1-\sin ^{2}(B) / B^{2} \text {. }
$$

The prediction of the circular variance versus bandwidth from this formula is the curve labeled $c=0$ in Figure 16. The explanation of the label is just below. The circular variance for such a tuning curve climbs monotonically with increasing bandwidth, with very low values of $V$ associated with very small values of bandwidth.

However, many neurons have orientation tuning curves with nonzero response at all orientations. For neurons with these less selective orientation tuning curves, Equation A1 relating circular variance to bandwidth does not predict the circular variance. Such a tuning curve can be approximated as the triangular tuning curve we used above, plus a DC response at all orientations, as depicted in Figure 15. One can derive a more general formula for the relationship between such an orientation tuning curve and its circular variance as follows. If a tuning curve is not zero outside the tuning band but instead has a baseline response $r_{0}$ at all orientations and a peak response $r_{0}+r_{p}$, then here is the

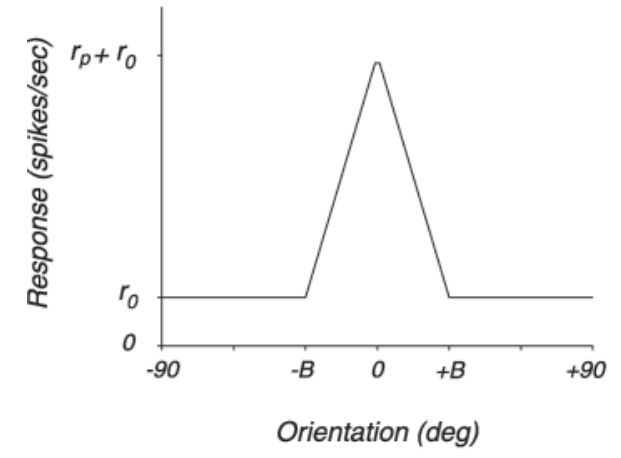

Figure 15. Diagram model of orientation tuning curves. The tuning curve of a model neuron that has a triangular-shaped tuning curve and also (possibly) a constant baseline response added to it at all orientations. This tuning curve is characterized by the parameters $B$, the intersection of the sloping portion of the tuning curve with the flat level portion, $r_{0}$, the level of constant response, and $r_{p}$, the height of the peak response above the constant level. 


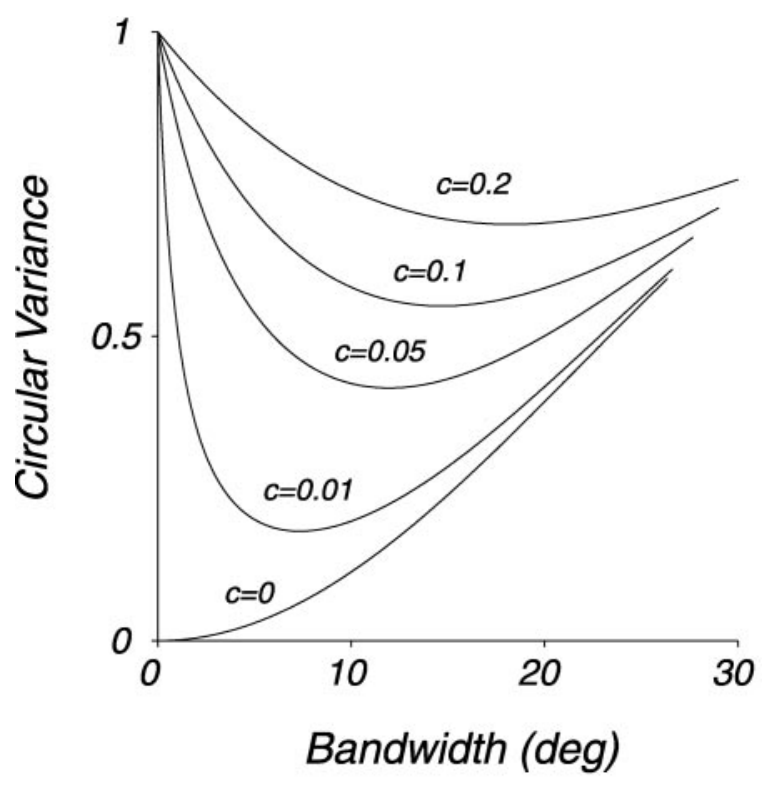

Figure 16. Relationship between orientation bandwidth and circular variance for model neurons. This is the illustration of the calculations in Appendix that show how circular variance and bandwidth are related for a range of different model tuning curves, with the parameter $c=r_{0} / r_{p}$ taking on the values $0,0.01,0.05,0.1$, and 0.2 .

relationship between the circular variance $V$, the parameter $B$ defined as above, and the ratio between the orthogonal and preferred responses $c=r_{0} / r_{p}$ :

$$
V=1-\Omega \sin ^{2}(B) \text { where } \Omega=1 /\left(B^{2}+2 \pi B c\right) .
$$

This formula reduces to the previous case when $c=0$. This is calculated as above, with the only difference that the denominator of the fraction for the resultant term is in this case $r_{p} B+2 \pi r_{0}$. To plot circular variance versus bandwidth as defined in this paper, we must calculate the bandwidth as well. The bandwidth at $1 / \sqrt{2}$ height is given by $B W=B(1+c)(1-1 / \sqrt{2})$.

Some sample functions relating circular variance and bandwidth are graphed in Figure 16 for different values of $c(c=0$, $0.01,0.05,0.1$, and 0.2 ). It can be seen that, when there is a significant wide angle response, even when it is small as in the $c=$ 0.05 case, the circular variance is much higher for a given bandwidth than is the case when $c=0$. This can explain why there are so many points in Figure 3 that have small bandwidths but relatively high circular variance.

\section{REFERENCES}

Anderson JS, Lampl I, Gillespie DC, Ferster D (2000) The contribution of noise to contrast invariance of orientation tuning in cat visual cortex. Science 290:1968-1972.

Batschelet E (1981) Circular statistics in biology. London: Academic.

Ben-Yishai R, Bar-Or RL, Sompolinsky H (1995) Theory of orientation tuning in visual cortex. Proc Natl Acad Sci USA 92:3844-3848.

Blasdel GG, Fitzpatrick D (1984) Physiological organization of layer 4 in macaque striate cortex. J Neurosci 4:880-895.

Bonds AB (1989) Role of inhibition in the specification of orientation selectivity of cells in the cat striate cortex. Vis Neurosci 2:41-55.

Carandini M, Ringach DL (1997) Predictions of a recurrent model of orientation selectivity. Vision Res 37:3061-3071.

Chance FS, Nelson SB, Abbott LF (1999) Complex cells as cortically amplified simple cells. Nat Neurosci 2:277-282.

Chapman B, Stryker MP (1993) Development of orientation selectivity in the ferret visual cortex and the effects of deprivation. J Neurosci 12:5251-5262.
DeValois RL, Yund EW, Hepler N (1982) The orientation and direction selectivity of cells in macaque visual cortex. Vision Res 22:531-544.

Ding Y, Casagrande VA (1997) The distribution and morphology of LGN K pathway axons within the layers and CO blobs of owl monkey V1. Vis Neurosci 14:691-704.

Ding Y, Casagrande VA (1998) Synaptic and neurochemical characterization of parallel pathways to the cytochrome oxidase blobs of primate visual cortex. J Comp Neurol 391:429-443.

Douglas RJ, Koch C, Mahowald M, Martin KC, Suarez HH (1995) Recurrent excitation in neocortical circuits. Science 269:981-985.

Dragoi V, Rivadulla C, Sur M (2001) Foci of orientation plasticity in visual cortex. Nature 411:80-86.

Enroth-Cugell C, Robson JG (1966) The contrast sensitivity of the retinal ganglion cells of the cat. J Physiol (Lond) 187:517-552.

Ferster D (1988) Spatially opponent excitation and inhibition in simple cells of the cat visual cortex. J Neurosci 8:1172-1180.

Ferster D, Miller KD (2000) Neural mechanisms of orientation selectivity in the visual cortex. Annu Rev Neurosci 23:441-471.

Fitzpatrick D, Lund JS, Blasdel GG (1985) Intrinsic connections of macaque striate cortex: afferent and efferent connections of lamina $4 \mathrm{c}$. J Neurosci 5:3329-3349.

Gegenfurtner KR, Kiper DC, Fenstemaker SB (1996) Processing of color, form and motion in macaque V2. Vis Neurosci 13:161-172.

Hawken MJ, Parker AJ (1984) Contrast sensitivity and orientation selectivity in lamina IV of the striate cortex of old world monkeys. Exp Brain Res 54:367-372.

Hawken MJ, Parker AJ, Lund JS (1988) Laminar organization and contrast sensitivity of direction-selective cells in the striate cortex of the old world monkey. J Neurosci 8:3541-3548.

Hendrickson AE, Wilson JR, Ogren MP (1978) The neuroanatomical organization of pathways between the dorsal lateral geniculate nucleus and visual cortex in old world and new world primates. J Comp Neurol 182:123-136.

Hendry SH, Yoshioka T (1994) A neurochemically distinct third channel in the macaque dorsal lateral geniculate nucleus. Science 264:575-577.

Hubel DH, Wiesel TN (1962) Receptive fields, binocular interaction and functional architecture of cat's visual cortex. J Physiol (Lond) 160:106-154.

Hubel DH, Wiesel TN (1968) Receptive fields and functional architecture of monkey striate cortex. J Physiol (Lond) 195:215-245.

Johnson EN, Hawken MJ, Shapley R (2001) The spatial transformation of color in the primary visual cortex of the macaque monkey. Nat Neurosci 4:409-416

Jones L, Palmer LA (1987) An evaluation of the two-dimensional Gabor filter model of simple receptive fields in cat striate cortex. J Neurophysiol 58:1233-1258.

Lachica EA, Beck PD, Casagrande VA (1992) Parallel pathways in macaque monkey striate cortex: anatomically defined columns in layer III. Proc Natl Acad Sci USA 89:3566-3570.

Leventhal AG, Thompson KG, Liu D, Zhou Y, Ault SJ (1995) Concomitant sensitivity to orientation, direction, and color of cells in layers $\sim 2$, 3 , and 4 of monkey striate cortex. J Neurosci 15:1808-1818.

Levick WR, Thibos LN (1982) Analysis of orientation bias in cat retina. J Physiol (Lond) 329:243-261.

Lund JS (1988) Anatomical organization of macaque monkey striate visual cortex. Annu Rev Neurosci 11:253-288.

Maffei L, Fiorentini A (1973) The visual cortex as a spatial frequency analyser. Vision Res 13:1255-1267.

Mardia KV (1972) Statistics of directional data. London: Academic.

McLaughlin D, Shapley R, Shelley M, Wielaard DJ (2000) A neuronal network model of macaque primary visual cortex (V1): orientation selectivity and dynamics in the input layer $4 \mathrm{C} \alpha$. Proc Natl Acad Sci USA 97:8087-8092.

Mechler F, Ringach DL (2002) On the classification of simple and complex cells. Vision Res 42:1017-1033.

Merrill EG, Ainsworth A (1972) Glass-coated platinum-plated tungsten microelectrodes. Med Biol Eng 10:662-672.

Movshon JA, Thompson ID, Tolhurst DJ (1978) Spatial summation in the receptive fields of simple cells in the cat's striate cortex. J Physiol (Lond) 283:53-77.

Rabiner LR, Gold B (1975) Theory and application of digital signal processing. Upper Saddle River, NJ: Prentice Hall.

Ringach DL, Hawken MJ, Shapley R (1997) Dynamics of orientation tuning in macaque primary visual cortex. Nature 387:281-284.

Ringach DL, Bredfeldt CE, Hawken MJ, Shapley R (2002) Suppression of neural responses to nonoptimal stimuli correlates with tuning selectivity in macaque V1. J Neurophysiol 87:1018-1027.

Sato H, Katsuyama N, Tamura H, Hata Y, Tsumoto T (1996) Mechanisms underlying orientation selectivity of neurons in the primary visual cortex of the macaque. J Physiol (Lond) 494:757-771.

Sceniak MP, Hawken MJ, Shapley RM (2001) Visual spatial characterization of macaque V1 neurons. J Neurophysiol 85:1873-1887.

Schiller PH, Finlay BL, Volman SF (1976) Quantitative studies of singlecell properties in monkey striate cortex. II. Orientation specificity and ocular dominance. J Neurophysiol 39:1320-1333. 
Sclar G, Freeman RD (1982) Orientation selectivity in the cat's striate cortex is invariant with stimulus contrast. Exp Brain Res 46:457-461.

Sillito AM (1977) Inhibitory processes underlying the directional specificity of simple, complex and hypercomplex cells in the cat's visual cortex. J Physiol (Lond) 271:699-720.

Skottun BC, DeValois RL, Grosof DH, Movshon JA, Albrecht DG, Bonds AB (1991) Classifying simple and complex cells on the basis of response modulation. Vision Res 31:1079-1086.

Somers DC, Nelson SB, Sur M (1995) An emergent model of orientation selectivity in cat visual cortical simple cells. J Neurosci 15:5448-5465.

Sompolinsky H, Shapley R (1997) New perspectives on the mechanisms for orientation selectivity. Curr Opin Neurobiol 7:514-522.

Swindale NV (1998) Orientation tuning curves: empirical description and estimation of parameters. Biol Cybern 78:45-56.
Troyer TW, Krukowski AE, Priebe NJ, Miller KD (1998) Contrastinvariant orientation tuning in cat visual cortex: thalamocortical input tuning and correlation-based intracortical connectivity. J Neurosci 18:5908-5927.

Wielaard DJ, Shelley M, McLaughlin D, Shapley R (2001) How simple cells are made in a nonlinear network model of the visual cortex. J Neurosci 21:5203-5211.

Wörgötter F, Eysel UT (1987) Quantification and comparison of cell properties in cat's striate cortex determined by different types of stimuli. Biol Cybern 57:349-355.

Yabuta NH, Callaway EM (1998) Functional streams and local connections of layer $4 \mathrm{C}$ neurons in primary visual cortex of the macaque monkey. J Neurosci 18:9489-9499.

Zhou H, Friedman HS, von der Heydt R (2000) Coding of border ownership in monkey visual cortex. J Neurosci 20:6594-611. 